\title{
Delay-Dependent Robust Stability of Time Delay Systems
}

\author{
Frédéric Gouaisbaut and Dimitri Peaucelle \\ LAAS-CNRS \\ 7, av. du colonel Roche, 31077 Toulouse, FRANCE \\ Email: \{gouaisbaut, peaucelle\}@laas.fr
}

September 5, 2005

\begin{abstract}
In this note, we provided an improved way of constructing a Lyapunov-Krasovskii functional for a linear time delay system. This technique is based on the reformulation of the original system and a discretization scheme of the delay. A hierarchy of Linear Matrix Inequality based results with increasing number of variables is given and is proved to have convergence properties in terms of conservatism reduction. Examples are provided which show the effectiveness of the proposed conditions.

Keywords: Linear time delay systems, Stability, Robustness
\end{abstract}

\section{Introduction}

During the last decades, stability of linear time delay systems have attracted a lot of attention, see (Moon et al., 2001; Park, 1999; Xu and Lam, 2005; Fridman and Shaked, 2002a) and references therein. The main approach relies on the use of a Lyapunov Krasovskii functional or a Lyapunov Razumikhin function. It leads to the so called delay dependent criteria which are expressed in terms of LMIs (linear matrix inequalities) and then easily solved using dedicated solvers. Generally, all these approach have to tackle with two main difficulties. The first one is the choice of the model transformation which is closely related to a choice of Lyapunov Krasovskii functional, see (Kolmanovskii and Richard, 1999) for a complete classification. The second problem lies on the bound of some cross terms which appears in the derivative of the Lyapunov functional, see (Park, 1999; Moon et al., 2001; Gu et al., 2003). The present paper brings a contribution to the first issue: by appropriate redundant modeling it introduces new types of Lyapunov Krasovskii functionals.

The methodology may be seen as similar to that in (Peaucelle et al., 2005) and (Ebihara et al., 2005). In these papers, parameter-dependent Lyapunov functions for robust analysis are exhibited by means of redundant system modeling using higher order times derivatives of the state. Most efficient for robustness problems, this approach is adapted here for time-delay systems. It is shown that introducing redundant 
differential equations shifted in time by a fractions of the time-delay allows to build new Lyapunov Krasovskii functionals that reduce the conservatism in searching for the maximal delay such that the system is asymptotically stable. As in formulated in $(\mathrm{Gu}$ et al., 2003, page 165) the present results are part of the implicit model transformation based methods.

An important feature of the present contribution is to build an infinite sequence of Lyapunov functionals and associated delay-dependent problems. Each problem of the sequence corresponds to a choice of an integer $r$ that defines the discretization of the delay in $r$ intervals of same length. For growing discretizations the problems are shown to have conservatism reduction properties. The building of sequences of conservative problems with convergence properties can also be found in (Bliman, 2002) and (Gu, 1997; Gu, 2001). In the first paper, the key idea is quite similar to ours but amounts to taking multiples of the delay while we discretize the delay. Moreover, the results of (Bliman, 2002) are relevant for delay-independent stability while we consider the delay-dependent case. As for the discretization scheme of $\mathrm{Gu}$, a detailed comparison is needed and it could not find its place in the present paper due to space limitations. But note that similarities exist (constant matrices of the Lyapunov functional on each discretization interval) as well as differences (we exhibit non integrated quadratic terms that depend on discretized values of the state).

All results are formulated in terms of Linear Matrix Inequalities (LMIs) and a particular attention is paid to formulating these results the most efficiently, that is, without introducing extra useless decision variables. In this, we follow methodologies based on Finsler lemma (Skelton et al., 1998) known to be very effective in robust control (De Oliveira and Skelton, 2001) and that has been already used for the study of time delay systems in the delay independent case (Castelan et al., 2003) and in the delay dependent case (Suplin et al., 2004)). As in these papers, we demonstrate that the approach is relevant not only for stability analysis of perfectly known models, but easily extends to robust stability analysis. Two such extensions are exposed: one in the quadratic stability framework, that is with Lyapunov functionnals that do not depend on the uncertain parameters; and the second taking advantage of parameter-dependent Lyapunov functionals.

The paper is organized as follows. In section 2, we derive a first conservative result for delay-dependent stability analysis. Although it is derived by means of known techniques, the result is totally new at our knowledge. Methodology for extension to robust analysis close this section. Then, in section 3 we expose the first step of our discretization scheme and prove that is does reduce the conservatism at the expense of an augmentation of the number of decision variables. The following section 4 gives the general result for a discretization of the delay in $r$ intervals. Section 5 is devoted to numerical experiments that illustrated the effectiveness of the approach.

Notations: For a two symmetric matrices, $A$ and $B, A>(\geq) B$ means that $A-$ $B$ is (semi-) positive definite. $A^{T}$ denotes the transpose of $A$. $1_{n}$ and $0_{m, n}$ denote the respectively the identity matrix of size $n$ and null matrix of size $n \times n$. If the context allows it the dimensions of these matrices are often omitted. For a given matrix $B \in \mathbb{R}^{m \times n}$ such that $\operatorname{rank}(B)=r$, we define $B^{\perp} \in \mathbb{R}^{n \times(n-r)}$ the right orthogonal complement of $B$ by $B B^{\perp}=0$ and $B^{\perp} B^{\perp T}>0$. The notation diag is used for block 
diagonal matrices:

$$
\operatorname{diag}(A, B, C)=\left[\begin{array}{ccc}
A & 0 & 0 \\
0 & B & 0 \\
0 & 0 & C
\end{array}\right]
$$

The Kronecker product of matrices is denoted $\otimes$ and is such that $1_{2} \otimes A=\operatorname{diag}(A, A)$, $1_{3} \otimes A=\operatorname{diag}(A, A, A)$.

\section{A first result on Stability}

Consider the following time delay system:

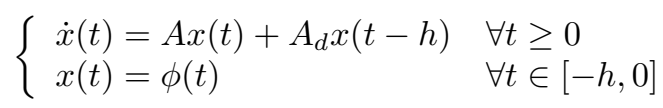

where $x(t) \in \mathbb{R}^{n}$ is the instantaneous state, $\phi$ is the initial condition and $A, A_{d} \in \mathbb{R}^{n \times n}$ are known constant matrices. $x_{t}$ is the state of the system:

$$
x_{t}(.):\left\{\begin{aligned}
{[-h, 0] } & \rightarrow R^{n} \\
\theta & \mapsto x_{t}(\theta)=x(t+\theta)
\end{aligned}\right.
$$

and we denote $\sigma_{\phi}$ the solution to the differential equation with initial conditions $\phi$. The following theorem gives a first result on the delay dependent stability for system (1).

Theorem 1 The system (1) is asymptotically stable for any delay $h$ such that $0 \leq h \leq$ $h_{m}$ if there exists $P>0, Q>0, R>0$ of appropriate dimensions satisfying the following LMI

$$
\begin{aligned}
& {\left[\begin{array}{rr}
A^{T} P+P A^{T}+Q & P A_{d}^{T} \\
A_{d}^{T} P & -Q
\end{array}\right]} \\
& h_{m}\left[\begin{array}{c}
A^{T} \\
A_{d}^{T}
\end{array}\right] R\left[\begin{array}{c}
A^{T} \\
A_{d}^{T}
\end{array}\right]^{T}-\frac{1}{h_{m}}\left[\begin{array}{c}
1 \\
-1
\end{array}\right] R\left[\begin{array}{c}
1 \\
-1
\end{array}\right]^{T}<0
\end{aligned}
$$

Proof : Define the following Lyapunov-Krasovskii functional for system (1):

$$
\begin{aligned}
& V\left(x_{t}\right)=x^{T}(t) P x(t)+ \\
& \int_{t-h}^{t} x^{T}(\theta) Q x(\theta) d \theta+\int_{t-h}^{t} \int_{s}^{t} \dot{x}^{T}(\theta) R \dot{x}(\theta) d \theta d s
\end{aligned}
$$

Remark that since $P, Q, R>0$, we can conclude that for some $\epsilon>0$, the LyapunovKrasovskii functional condition $V\left(x_{t}\right) \geq \epsilon\left\|x_{t}(0)\right\|$ is satisfied (see (Gu et al., 2003)). The derivative along the trajectories of (1) leads to the following equality :

$$
\begin{aligned}
\dot{V}\left(x_{t}\right) & =2 x^{t}(t) P \dot{x}(t)+x^{T}(t) Q x(t) \\
& -x^{T}(t-h) Q x(t-h)+h \dot{x}^{T}(t) R \dot{x}(t) \\
& -\int_{t-h}^{t} \dot{x}^{T}(\theta) R \dot{x}(\theta) d \theta
\end{aligned}
$$


Using the Jensen's inequality (see (Gu et al., 2003) and references therein), the last term can be bounded as follows :

$$
-\int_{t-h}^{t} \dot{x}^{T}(\theta) R \dot{x}(\theta) d \theta<-z^{T}(t) \frac{R}{h} z(t)
$$

where $z(t)=\int_{t-h}^{t} \dot{x}(\theta) d \theta=x(t)-x(t-h)$. Therefore we get $\dot{V}\left(x_{t}\right)<\zeta^{T} \mathcal{M}(h) \zeta$ with

$$
\zeta=\left(\begin{array}{l}
\dot{x}(t) \\
x(t) \\
x(t-h) \\
z(t)
\end{array}\right), \mathcal{M}(h)=\left[\begin{array}{cccc}
h R & P & 0 & 0 \\
P & Q & 0 & 0 \\
0 & 0 & -Q & 0 \\
0 & 0 & 0 & -\frac{1}{h} R
\end{array}\right]
$$

Furthermore, using the extended variable $\zeta$, system (1) with the extra variable $z(t)$ can be rewritten as $B \zeta=0$ where $B=\left[\begin{array}{cccc}1 & -A & -A_{d} & 0 \\ 0 & -1 & 1 & 1\end{array}\right]$. The original system (1) is asymptotically stable if for all $\zeta$ such that $B \zeta=0$, the inequality $\zeta^{T} \mathcal{M}(h) \zeta<0$ holds. Using Finsler lemma (Skelton et al., 1998), this is equivalent to $B^{\perp T} \mathcal{M}(h) B^{\perp}<0$, where $B^{\perp}$ is a right orthogonal complement of $B$. Furthermore, it can be easily seen that $\mathcal{M}(h) \leq \mathcal{M}\left(h_{m}\right)$ if $h<h_{m}$, i.e. if asymptotic stability is proved using this result for a delay $h_{m}$ then it also holds for any smaller delay.

An admissible value of $B^{\perp}$ is the following:

$$
B^{\perp}=\left[\begin{array}{cccc}
A^{T} & 1 & 0 & 1 \\
A_{d}^{T} & 0 & 1 & -1
\end{array}\right]^{T}
$$

Simple calculations show that $B^{\perp T} \mathcal{M}\left(h_{m}\right) B^{\perp}<0$ is equivalent to (2), which concludes the proof.

Remark 1 Instead of using the orthogonal complement of B, Finsler lemma also states that condition $B^{\perp T} \mathcal{M} B^{\perp}<0$ is equivalent to the existence of some $F \in$ $\mathbb{R}^{2 n \times 4 n}$ such that the LMI $\mathcal{M}+F B+B^{T} F^{T}<0$ holds. Creating such additional variable $F$ is trivially useless for the considered case: it only increases the number of variables and constraints in the LMI problem without reducing anyhow the conservatism of the approach. But as demonstrated in (Peaucelle and Gouaisbaut, 2005) and many others, such additional 'slack variables' are of major interest for robust analysis purpose.

Assume that the system matrices are not precisely known but belong to a given convex set of finitely many vertices (also called polytope of matrices). The set of possible values of the matrices may be parameterized using barycentric coordinates as:

$$
\left[\begin{array}{ll}
A(\lambda) & A_{d}(\lambda)
\end{array}\right]=\sum_{i=1}^{N} \lambda_{i}\left[\begin{array}{ll}
A^{[i]} & A_{d}^{[i]}
\end{array}\right]
$$

where $\lambda_{i} \geq 0$ are positive and their sum is one: $\sum_{i=1}^{N} \lambda_{i}=1$. The matrices with subscripts ${ }^{[i]}$ are called the vertices. Based on the result of Theorem 1, proving robust asymptotic stability for the resulting uncertain system can be achieved by finding 
parameter dependent matrices $P(\lambda), Q(\lambda)$ and $R(\lambda)$ such that (2) holds for all admissible values of $\lambda$. This may not be done in general due to the infinite number of admissible values for $\lambda$, but two relaxations may be stated.

Theorem 2 The uncertain system combining (1) and (6) is robustly asymptotically stable if any of the following LMI conditions hold

(i) There exist $P>0, Q>0, R>0$ unique over all uncertainties such that the $L M I(2)$ holds for all $N$ vertices.

(ii) There exist polytopic matrices

$$
\begin{gathered}
P(\lambda)=\sum_{i=1}^{N} \lambda_{i} P^{[i]} \\
Q(\lambda)=\sum_{i=1}^{N} \lambda_{i} Q^{[i]}, R(\lambda)=\sum_{i=1}^{N} \lambda_{i} R^{[i]}
\end{gathered}
$$

with positive definite vertices $\left(P^{[i]}>0, \ldots\right)$ and a unique $F$ such that the LMIs

$$
\mathcal{M}^{[i]}+F B^{[i]}+B^{[i]^{T}} F^{T}<0
$$

hold for all $N$ vertices.

Moreover, condition (ii) is allways satified if (i) holds.

The proof is omitted for space limitation reasons and because it is now classical in the robust analysis context. The purpose of Theorem 2 is to illustrate that all results of the present paper can be easily extended to the robust analysis of polytopic uncertain systems. Moreover, the extensions correspond to two major approaches of robust control theory: (i) corresponds to the quadratic stability framework in which the matrices defining the Lyapunov functional are unique over all uncertainties; (ii) corresponds to the slack variables framework that first allowed to search for polytopic parameterdependent Lyapunov functionals. See for example (Peaucelle et al., 2000) for details on this subject.

In the following, robustness issues will no longer be detailed, but similar results may be easily derived.

\section{A first step to a discretization scheme}

To our knowledge the result of Theorem 1 is a new formulation of existing equivalent results. The detailed comparison is left for a specific paper (Gouaisbaut and Peaucelle, 2006). Here, we aim at developing further the methodology used in the previous section to derive less conservative results.

The key idea is that since Theorem 1 proves asymptotic stability for all delays $0 \leq h \leq h_{m}$, then this property should also hold for $h_{m} / 2$. Introducing the half delay into the system should improve the knowledge on the system and hence the results.

Theorem 3 System (1) is asymptotically stable for any delay $h$ such that $0 \leq h \leq h_{m}$ if there exists $P_{2}>0, Q_{21} \geq 0, Q_{22}>0, R_{21} \geq 0, R_{22}>0 \in \mathbb{R}^{2 n \times 2 n}$ satisfying the following LMI :

$$
B_{2}^{\perp T} \mathcal{M}_{2}\left(h_{m}\right) B_{2}^{\perp}<0
$$


where $B_{2}^{\perp}$ is an orthogonal complement of:

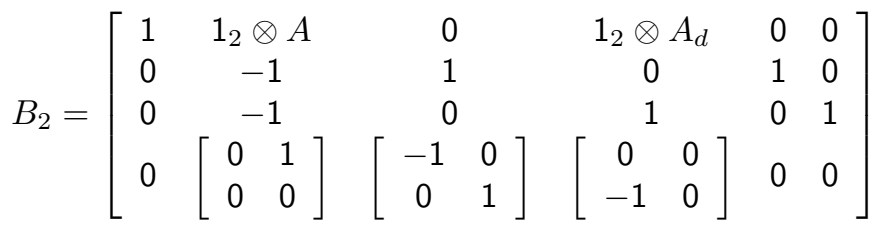

and $\mathcal{M}_{2}(h)=$

$$
\left[\begin{array}{cccc}
\frac{h}{2} R_{21}+h R_{22} & P_{2} & 0 & 0 \\
P_{2} & Q_{21}+Q_{22} & 0 & 0 \\
0 & 0 & -\mathcal{Q}_{2} & 0 \\
0 & 0 & 0 & -\mathcal{R}_{2}
\end{array}\right]
$$

with

$$
\mathcal{Q}_{2}=\operatorname{diag}\left(Q_{21}, Q_{22}\right), \mathcal{R}_{2}=\operatorname{diag}\left(\frac{2}{h} R_{21}, \frac{1}{h} R_{22}\right)
$$

Proof : Consider system (1). It may as well be written for any $\theta$ such that $0 \leq \theta \leq h$ as follows

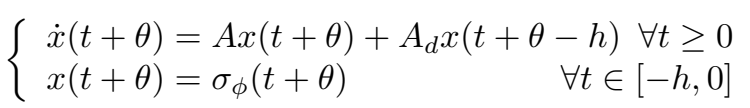

where $\sigma_{\phi}$ is the solution to (1). Choose $\theta=\frac{h}{2}$ and consider the artificially augmented system:

$$
\left\{\begin{array}{l}
\dot{x}\left(t+\frac{h}{2}\right)=A x\left(t+\frac{h}{2}\right)+A_{d} x\left(t-\frac{h}{2}\right) \\
\dot{x}(t)
\end{array}\right.
$$

with accordingly defined initial conditions. Introducing the augmented instantaneous state

$$
x_{2}(t)=\left(\begin{array}{c}
x\left(t+\frac{h}{2}\right) \\
x(t)
\end{array}\right)
$$

the differential equations (9) write as:

$$
\begin{array}{r}
\dot{x}_{2}(t)=\left(1_{2} \otimes A\right) x_{2}(t)+0 x_{2}\left(t-\frac{h}{2}\right) \\
+\left(1_{2} \otimes A_{d}\right) x_{2}(t-h)
\end{array} .
$$

Define the extended variable

$$
\zeta_{2}=\left(\begin{array}{l}
\dot{x}_{2}(t) \\
x_{2}(t) \\
x_{2}\left(t-\frac{h}{2}\right) \\
x_{2}(t-h) \\
x_{2}(t)-x_{2}\left(t-\frac{h}{2}\right) \\
x_{2}(t)-x_{2}(t-h)
\end{array}\right) .
$$

Taking into account all interactions between the elements of $\zeta_{2}$, the system (9) can be modeled as constrained to the null space of $B_{2}$, that is $B_{2} \zeta_{2}(t)=0$. 
We now consider the following Lyapunov-Krasovskii functional:

$$
\begin{aligned}
V_{2}\left(x_{2 t}\right)= & x_{2}^{T}(t) P_{2} x_{2}(t) \\
& +\sum_{i=1}^{2} \int_{t-\frac{i h}{2}}^{t} x_{2}^{T}(\theta) Q_{2 i} x_{2}(\theta) d \theta \\
& +\sum_{i=1}^{2} \int_{t-\frac{i h}{2}}^{t} \int_{s}^{t} \dot{x}_{2}^{T}(\theta) R_{2 i} \dot{x}_{2}(\theta) d \theta d s
\end{aligned}
$$

Using the same idea developed in the proof of Theorem 1, we get that the derivative of (11) is such that:

$$
\dot{V}\left(x_{2 t}\right) \leq \zeta_{2}^{T} \mathcal{M}_{2} \zeta_{2}
$$

Using Finsler lemma, and similar arguments as in the proof of Theorem 1, conditions (7) imply that system (9) is asymptotically stable. For any initial conditions, the whole state $x_{2 t}$ converges asymptotically to zero. Its components $x_{t}$ converge as well. The initial system (1) is asymptotically stable.

For deriving the result of Theorem 3 we have taken advantage of the implicit model transformation (Gu et al., 2003, page 165) that extends the information on the state $x_{t}$ to an interval of width $2 h$. The functional (11) can therefore be seen as a new Lyapunov functional for (1) with an implicitly augmented information on the state.

At the expense of increasing the number of decision variables and constraints, Theorem 3 gives a new conservative result for the same problem as Theorem 1. More precisely the number of decision variables has been increased from $\frac{3}{2} n(n+1)$ in Theorem 1 to $5 n(2 n+1)$ in Theorem 3. This should go along with a reduction of the conservatism to be acceptable and indeed we get the following result.

Proposition 1 Let $h_{m}$ the maximum allowed solution of the problem (2), then $h_{m}$ is also a solution of (7).

Proof: Let $h_{m}$ and $P, Q, R$ solution of problem (2), and define

$$
\begin{gathered}
P_{2}=\left[\begin{array}{ll}
P & 0 \\
0 & P
\end{array}\right], Q_{22}=\left[\begin{array}{cc}
Q & 0 \\
0 & Q
\end{array}\right], R_{22}=\left[\begin{array}{cc}
R & 0 \\
0 & R
\end{array}\right] \\
Q_{21}=0, R_{21}=0 .
\end{gathered}
$$

Take the right orthogonal of $B_{2}$ such as

$$
B_{2}^{\perp}=\left[\begin{array}{cccccc}
1_{2} \otimes A^{T} & 1 & {\left[\begin{array}{ll}
0 & 0 \\
1 & 0
\end{array}\right]} & 0 & {\left[\begin{array}{cc}
1 & 0 \\
-1 & 1
\end{array}\right]} & 1 \\
1_{2} \otimes A_{d}^{T} & 0 & {\left[\begin{array}{ll}
0 & 1 \\
0 & 0
\end{array}\right]} & 1 & {\left[\begin{array}{cc}
0 & -1 \\
0 & 0
\end{array}\right]} & -1
\end{array}\right]^{T} .
$$

It appears that inequality (7) is nothing but (2) repeated twice on the diagonal.

\section{The general case}

In the previous section a new result, less conservative than the first one, is obtained by means of augmentation of the state variables introducing a half delay. This methodology is now generalized by discretizing $r$ times the interval $[-h 0]$. 
Given a strictly positive integer $r$, we introduce the followings reals:

$$
\left\{\begin{array}{l}
h_{0}=0 \\
h_{i}=\frac{i h}{r} \forall i \in\{1, \ldots, r\}
\end{array}\right.
$$

where $h$ is the delay of system (1). We have the following property :

$$
\left\{\begin{array}{l}
h_{r}=h \\
h_{i+j}=h_{i}+h_{j}, \forall(i, j) \in\{1, \ldots, r\}
\end{array}\right.
$$

Using equation (8) with $\theta=\left\{h_{0} \ldots h_{r-1}\right\}$, original system (1) is equivalent to :

$$
\dot{x}_{r}(t)=\sum_{i=0}^{r} \mathcal{A}_{d i} x_{r}\left(t-h_{i}\right)
$$

with the augmented state:

$$
x_{r}(t)=\left(\begin{array}{l}
x\left(t+h_{r_{1}}\right) \\
\vdots \\
x\left(t+h_{1}\right) \\
x\left(t+h_{0}\right)
\end{array}\right) \in \mathbb{R}^{n r}
$$

and the augmented system matrices,

$$
\begin{gathered}
\mathcal{A}_{d 0}=1_{r} \otimes A, \mathcal{A}_{d r}=1_{r} \otimes A_{d}, \\
\mathcal{A}_{d i}=0_{n r}, \quad \forall i \in\{1, \ldots r-1\} .
\end{gathered}
$$

With these notations the next Theorem exposes the generalization of Theorem 3 to the case of $1 / r$ discretization of the delay.

Theorem 4 Let any positive integer $r$. System (1) is asymptotically stable for any delay $h$ such that $0 \leq h \leq h_{m r}$ if there exists $P_{r}>0, Q_{r i}>0, R_{r i}>0, \forall i \in\{1, \ldots, r\} \in$ $\mathbb{R}^{r n \times r n}$ satisfying the following $L M I$ :

$$
B_{r}^{\perp T} \mathcal{M}_{r}\left(h_{m}\right) B_{r}^{\perp}<0
$$

where $B_{r}^{\perp}$ is the orthogonal complement of $B_{r}=$

$$
\left[\begin{array}{cccccccccc}
1 & -\mathcal{A}_{d 0} & -\mathcal{A}_{d 1} & -\mathcal{A}_{d 2} & \ldots & -\mathcal{A}_{d r} & 0 & 0 & \ldots & 0 \\
0 & -1 & 1 & 0 & \ldots & 0 & 1 & 0 & \ldots & 0 \\
0 & -1 & 0 & 1 & \ldots & 0 & 0 & 1 & \ldots & 0 \\
\vdots & \vdots & \vdots & 0 & \ddots & 0 & \vdots & 0 & \ddots & 0 \\
0 & -1 & 0 & 0 & \ldots & 1 & 0 & 0 & \ldots & 1 \\
0 & E_{r 1} & -E_{r 2} & 0 & \ldots & \ldots & \ldots & \ldots & \ldots & 0 \\
0 & 0 & E_{r 1} & -E_{r 2} & 0 & \ldots & \ldots & \ldots & \ldots & 0 \\
\vdots & \vdots & 0 & \ddots & \ddots & 0 & \ldots & \ldots & \ldots & \vdots \\
0 & 0 & 0 & 0 & E_{r 1} & -E_{r 2} & 0 & 0 & 0 & 0
\end{array}\right]
$$


where

$$
\begin{gathered}
E_{r 1}=\left[\begin{array}{ll}
0_{(r-1) n, n} & 1_{(r-1) n}
\end{array}\right] \\
E_{r 2}=\left[\begin{array}{lll}
1_{(r-1) n} & 0_{(r-1) n, n}
\end{array}\right], \\
\mathcal{M}_{r}(h)=\left[\begin{array}{cccc}
\sum_{i=1}^{r} h_{i} R_{r i} & P_{r} & 0 & 0 \\
P_{r} & \sum_{i=1}^{r} Q_{r i} & 0 & 0 \\
0 & 0 & -\mathcal{Q}_{r} & 0 \\
0 & 0 & 0 & -\mathcal{R}_{r}
\end{array}\right]
\end{gathered}
$$

and

$$
\begin{aligned}
& \mathcal{Q}_{r}=\operatorname{diag}\left(Q_{r 1}, \ldots, Q_{r r}\right) \\
& \mathcal{R}_{r}=\operatorname{diag}\left(\frac{1}{h_{1}} R_{r 1}, \ldots, \frac{1}{h_{r}} R_{r r}\right)
\end{aligned}
$$

The proof follows the same lines as the proof of Theorem 3 and is therefore omitted for reasons of space limitation. For the same reasons the next Proposition is not proved. As for Proposition 1, it follows from the fact that a thinner discretization of the interval $\left[-h_{m} 0\right]$ reduces the conservatism as long as it includes the discretization to be compared.

Proposition 2 Let $r_{2}$ be a multiple of $r_{1}$ (i.e. $r_{2}=k r_{1}$ for some integer $k$ ) and let $h_{m r_{1}}$ be the maximum allowed solution of the problem (14) when $r=r_{1}$, then $h_{m r_{1}} \leq h_{m r_{2}}$ where $h_{m r_{2}}$ is the maximal allowable solution of (14) for $r=r_{2}$.

This proposition shows that the conservative relaxations of the time-delay analysis problem have converging properties when taking thinner discretizations. This improvement goes along with the augmentation of the numerical complexity. For the relaxation of order $r$ the number of decision variables is $\frac{1}{2}(1+2 r) r n(r n+1)$ and LMI constraint (14) is of dimension $2 r n \times 2 r n$.

Remark 2 Theorem 4 is formulated using matrices $\mathcal{A}_{d i}$ all set to zero for $i=\{1 \ldots r-$ $1\}$. These correspond to fictive influence of the dicretized delay on the system dynamics. A by product of this result is that using the same methodology it is possible to solve stability analysis of systems with multiple delays as long as the delays can be written as subdivisions of the largest one.

\section{Examples}

Example 1 Consider the time delay system (1) with

$$
A=\left[\begin{array}{cc}
-2 & 0 \\
0 & -0.9
\end{array}\right], A_{d}=\left[\begin{array}{cc}
-1 & 0 \\
-1 & -1
\end{array}\right]
$$

For this academic example many results were obtained in the literature. Table 1 summarizes these and compares them to the new results presented in the paper. $h_{\max }$ is the maximal allowable delay proved by each method and $n b$ vars. indicates the number of variables of the associated LMI problem. In all methods $h_{\max }$ is obtained by a line search. 
Table 1: Results for Example 1

\begin{tabular}{llr}
\hline Methods & $h_{\max }$ & nb vars. \\
\hline (Li and De Souza, 1997) & 0.8571 & 9 non LMI \\
(Niculescu et al., 1995) & 0.99 & 11 \\
(Moon et al., 2001) & 4.3588 & 16 \\
(Han, 2002) & 4.4721 & 9 or 18 \\
(Fridman, 2002) & 4.47 & 27 \\
(Xu and Lam, 2005) & 4.4721 & 17 \\
(Suplin et al., 2004) & 4.4721 & 38 \\
Theorem 1 & 4.4721 & 9 \\
Theorem 3 & 5.71 & 50 \\
Theorem 4 r=3 & 5.91 & 147 \\
Theorem 4 r=4 & 6.03 & 324 \\
Theorem 4 r=5 & 6.09 & 605 \\
Theoretical bound & 6.17 & $\infty$ \\
\hline
\end{tabular}

Remark 3 The numerical experiments of Table 1 show that Theorem 1 gives similar results to papers using descriptor system approach and bounding techniques from (Lee et al., 2004) and (Moon et al., 2001). Investigations to link all these results are developped in (Gouaisbaut and Peaucelle, 2006).

Example 2 Again an academic example is chosen for comparison with existing results. It corresponds to an uncertain time delay system with two vertices

$$
\begin{gathered}
A^{[1]}=\left[\begin{array}{ll}
0 & -0.54 \\
1 & -0.43
\end{array}\right], A^{[2]}=\left[\begin{array}{cc}
0 & 0.3 \\
1 & -0.5
\end{array}\right] \\
A_{d}^{[1,2]}=\left[\begin{array}{cc}
-0.1 & -0.35 \\
0 & 0.3
\end{array}\right]
\end{gathered}
$$

The robust versions of our results using methodology (ii) of Theorem 2 are applied and compared to existing results in Table 2.

Table 2: Results for Example 2

\begin{tabular}{ll}
\hline Methods & $h_{\max }$ \\
\hline (Fridman and Shaked, 2002b) & 0.782 \\
(Suplin et al., 2004) & 0.863 \\
Theorem 1 & 0.896 \\
Theorem 3 & 0.897 \\
\hline
\end{tabular}




\section{References}

Bliman, P.A. (2002). Lyapunov equation for the stability of linear delay systems of retarded and neutral type. IEEE Trans. Aut. Control 47(2), 327-335.

Castelan, E.B., i. Queinnec and S. Tarbouriech (2003). Sliding mode time-delay systems.. In: Proc. 4th IFAC Workshop on Time Delay Systems (TDS'03). Rocquencourt, France.

De Oliveira, M.C. and R.E. Skelton (2001). Stability tests for constrained linear systems. pp. 241-257. Lecture notes in control and information sciences. Springer. Berlin.

Ebihara, Y., D. Peaucelle, D. Arzelier and T. Hagiwara (2005). Robust performance analysis of linear time-invariant uncertain systems by taking higher-order timederivatives of the states. In: joint IEEE Conference on Decision and Control and European Control Conference. Seville, Spain.

Fridman, E. (2002). Stability of linear descriptor systems with delay: A lyapunovbased approach. Journal of Mathematical Analysis and Applications 273(1), 2444.

Fridman, E. and U. Shaked (2002a). A descriptor system approach to $h_{\infty}$ control of linear time-delay systems. IEEE Trans. Aut. Control 47(2), 253-270.

Fridman, E. and U. Shaked (2002b). An improved stabilization method for linear timedelay systems. IEEE Trans. Aut. Control 47(11), 1931-1937.

Gouaisbaut, F. and D. Peaucelle (2006). A note on the stability of time-delay systems. In: ROCOND. Toulouse. Submitted.

$\mathrm{Gu}$, K. (1997). Discretized LMI set in the stability problem of linear uncertain timedelay systems. Internat. J. Control 68, 923-934.

$\mathrm{Gu}$, K. (2001). Discretization schemes for lyapunov-krasovskii functionals in timedelay systems. Kybernetika 37(4), 479-504.

Gu, K., K.L. Kharitonov and J. Chen (2003). Stability of Time-Delay Systems. Control Engineering Series. Birkhauser. Boston USA.

Han, Q.L. (2002). Robust stability of uncertain delay-differential systems of neutral type. Automatica 38, 719-723.

Kolmanovskii, V.B. and J.P. Richard (1999). Stability of some linear systems with delay. IEEE Trans. Aut. Control 44(5), 984-989.

Lee, Y.S., Y.S. Moon and Park P. (2004). Delay-dependent robust $h_{\infty}$ control for uncertain systems with a state-delay. Automatica 40, 65-72.

Li, X. and C.E. De Souza (1997). Delay-dependent robust stability and stabilization of uncertain linear delay systems : A linear matrix inequality approach. IEEE Trans. Aut. Control 42(8), 1144-1148. 
Moon, Y. S., P. Park, W. H. Kwon and Y. S. Lee (2001). Delay-dependent robust stabilization of uncertain state-delayed systems. Int. J. Control 74, 1447-1455.

Niculescu, S., A. Trofino, J.M. Dion and L. Dugard (1995). Delay dependent stability of linear systems with delayed state: an LMI approach. In: IEEE Conf. on Decision an Control. New Orleans. pp. 1495-1496.

Park, P. (1999). A delay-dependent stability criterion for systems with uncertain timeinvariant delays. IEEE Trans. Aut. Control 44(4), 876-877.

Peaucelle, D. and F. Gouaisbaut (2005). Discussion on "parameter-dependent Lyapunov functions approach to stability analysis and design for uncertain systems with time-varying delay". European J. of Control 11(1), 69-70.

Peaucelle, D., D. Arzelier, O. Bachelier and J. Bernussou (2000). A new robust Dstability condition for real convex polytopic uncertainty. Systems \& Control Letters 40(1), 21-30.

Peaucelle, D., D. Henrion and D. Arzelier (2005). Quadratic separation for feedback connection of an uncertain matrix and an implicit linear transformation. In: 16th IFAC World Congress. Prague, Czech Republic.

Skelton, R.E., T. Iwazaki and K. Grigoriadis (1998). A unified Approach to Linear Control Design. Taylor and Francis series in Systems and Control.

Suplin, V., E. Fridman and U. Shaked (2004). A projection approach to $h_{\infty}$ control of time-delay systems. In: Proc. 43th IEEE CDC'04. Atlantis, Bahamas.

$\mathrm{Xu}, \mathrm{S}$. and J. Lam (2005). Improved delay-dependent stability criteria for time-delay systems. IEEE Trans. Aut. Control 50(3), 384-387. 